\title{
Multisensor Hybrid Dynamic Alliance Formation Problem Using Sensitive Particle-Based Dynamic Discrete PSO
}

\author{
Daozhi Wei, ${ }^{1}$ Zhaoyu Zhang ${ }^{(D},{ }^{1}$ Jiahao Xie, ${ }^{1}$ Liang fu Yao, ${ }^{2}$ and Ning Li $^{1}$ \\ ${ }^{1}$ Air and Missile Defense College, Air Force Engineering University, Xi'an 710051, China \\ ${ }^{2}$ Air and Missile Defense College, Unit 93682, Fengbo, Beijing 101300, China \\ Correspondence should be addressed to Zhaoyu Zhang; 3055574144@qq.com
}

Received 17 June 2021; Revised 9 November 2021; Accepted 17 November 2021; Published 9 December 2021

Academic Editor: Calogero Orlando

Copyright (c) 2021 Daozhi Wei et al. This is an open access article distributed under the Creative Commons Attribution License, which permits unrestricted use, distribution, and reproduction in any medium, provided the original work is properly cited.

In recent years, with the wide application and popularization of artificial intelligence algorithm in the field of multisensor information processing, it has been a research hotspot to solve the problem of sensor alliance formation in the battlefield environment by using multisensor cross-cueing technology. Based on the establishment of the multisensor hybrid dynamic alliance model and objective function, a multisensor cross-cueing algorithm based on dynamic discrete particle swarm optimization (DDPSO) with sensitive particles is proposed and a mechanism of "predict re-predict" is proposed in the process of sensor handover. Simulations have verified the good convergence effect and small detection error of multisensor cross-cueing technology in solving alliance formation problems. Meanwhile, compared with "measurement and then update" and "predict and update" mechanisms, the proposed mechanism is more suitable to the changing combat environment. At the same time, to some extent, it also shows that the artificial intelligence algorithm is more suitable for multisensor information processing.

\section{Introduction}

Artificial intelligence technology is currently at the forefront of academic innovation and more and more widely used in target detection and tracking $[1,2]$. However, only a single sensor cannot fully achieve early warning detection of targets, so it is necessary to use multisensor cooperation to achieve continuous high probability detection of targets [3-6]. An important way of cooperative detection is to build multisensor dynamic alliance $[7,8]$. The concept of dynamic alliance $[9,10]$ was first put forward in the manufacturing industry in the United States in order to improve production efficiency [11-13] and subsequently was introduced into wireless sensor networks by domestic scholars $[14,15]$. At present, most of the establishment of dynamic alliance is based on serial structure [16-20]. The main problem of this structure is low efficiency and easy-to-cause resource waste or excessive strain. The formation of multisensor dynamic alliance is mainly divided into two parts: formation and connection. The essence of multisensor alliance formation belongs to multisensor multitarget assignment problem. At present, there are many algorithms for multisensor multitarget assignment problem in the field of artificial intelligence at home and abroad, such as genetic algorithm [21], genetic algorithm optimization [22], particle swarm algorithm [23], and particle swarm optimization algorithm [22, 24-28], represented by particle swarm optimization, and a series of intelligence algorithms such as linear programming method [29] and auction algorithm [30]. Carlisle and Dozier [31] proposed an improved particle swarm optimization algorithm based on periodic iteration count resetting and environment change triggering resetting the particle's optimal position. In the experiment, the position and speed of the target are fixed, and the fitness function is simple, which cannot meet the requirements of the multisensor target detection model. Chen et al. [32] proposed particle swarm optimization (PSO) to solve the problem of alliance formation, but the algorithm did not consider the dynamic changes of the environment. Fan et al. [33] adopted discrete particle swarm optimization algorithm (DPSO) to realize alliance building, which proved the effectiveness of swarm intelligence algorithm to a certain extent but did not 
consider the consumption problem in alliance formation. Pang et al. [34] proposed the switching mechanism of "detection is update" when studying the switching mechanism of multisensor alliance. However, in practical application, the high-speed movement of the target will lead to target loss to a certain extent, resulting in low measurement accuracy affecting prediction. Fu et al. [35] proposed a switching mechanism of "predict is update," which is improved compared with the switching mechanism of "measure is update." However, dynamic changes of the battlefield environment are not taken into account, and there is a risk of target loss in the switching process.

In this paper, the application of multisensor cross-cueing technology in swarm intelligence is proposed to solve the formation and connection problem of multisensor hybrid dynamic alliance. According to the hybrid dynamic alliance model, a multisensor cross-cueing algorithm based on dynamic discrete particle swarm optimization (DPSO) with sensitive particles is designed and the effectiveness of swarm intelligence algorithm is verified by simulation analysis compared with the existing literature. At the same time, the mechanism of "predict and re-predict" is proposed to complete the task of alliance transfer and achieve stable detection of the target.

In this paper, we propose the application of improved swarm intelligence algorithm (DDPSO) in multisensor cross-cueing technology. In the first section, the mathematical model of multisensor dynamic alliance is constructed and the alliance detection model is proposed. Section 2 designs the detailed steps of multisensor crossprompt algorithm, Section 3 carries on the demonstration verification, and Section 4 summarizes the work of the whole paper and draws the conclusion.

\section{Multisensor Hybrid Dynamic Alliance Model}

2.1. Description of Hybrid Dynamic Alliance. The dynamic alliance model mainly refers to a temporary dynamic whole formed by several groups through negotiation among individuals in order to realize common interests and trigger by actual needs. However, the existing alliance structure is inefficient for knowing how to allocate sensors reasonably when the detection resources are limited. It is likely that many sensors will lose their targets because they cannot get or they miss the sensor alliance resources because of the time relationship, which will affect the detection effect and lead to the failure of the final task. Therefore, this paper introduces the hybrid multisensor dynamic alliance model to improve the detection accuracy and reduce the detection cost. Small detection errors can realize continuous detection and stable detection of targets [36].

In the model of multisensor hybrid dynamic alliance, it is assumed that each sensor has the ability of independent decision-making. At the same time, it can dynamically change according to the changes of the environment to give full play to the greatest advantage of the alliance and accomplish tasks efficiently. In the process of building hybrid dynamic alliance with multisensors, there are two main modes. One is that a sensor can join multiple sensor alliances or a task can be performed by multiple sensor alliances. The other is that a sensor alliance can handle multiple tasks at the same time. The specific build process is shown in Figure 1.

2.2. Target Motion Model. Suppose that the state of the target is $\mathbf{X}_{k}=[x(k), y(k), \dot{x}(k), \dot{y}(k)]^{T}$, the state transition matrix of the target is $\mathbf{X}_{k+1}=\Phi \mathbf{X}_{k}+\Gamma \mathbf{W}_{k}$, and the observation equation of the sensor is $\mathbf{Z}_{k}=\mathbf{H} \mathbf{X}_{k}+\mathbf{V}_{k}$, where $\Phi$ and $\Gamma$ are the state transition matrix of the target and the distribution matrix of the process noise, $\mathbf{W}_{k}$ and $\mathbf{V}_{k}$ are the system noise and the observation noise, $\mathbf{W}_{k}$ 's covariance matrix is $\mathbf{Q}_{k}$, and $\mathbf{V}_{k}$ 's covariance matrix is $\mathbf{R}_{k}$. The initial sensor alliance formed when the sensor tracks the target at a certain time $k$ is $s_{\text {initial }}=\left\{s_{1}, s_{2}, \cdots, s_{m}\right\}$.

In the process of target detection, the Federal Filtering (FKF) algorithm is used to estimate the state of the target. Four typical processes of FKF algorithm are as follows:

(1) Information distribution process:

$$
\begin{aligned}
& \mathbf{Q}_{i}(k)=\beta_{i}^{-1} \mathbf{Q}(k), \\
& \mathbf{P}_{i}(k)=\beta_{i}^{-1} \mathbf{P}_{g}(k), \\
& \mathbf{X}_{i}(k)=\mathbf{X}_{g}(k) .
\end{aligned}
$$

(2) Information time update:

$$
\begin{aligned}
\widehat{\mathbf{X}}_{i}(k+1 \mid k)= & \boldsymbol{\Phi}_{i}(k+1, k) \widehat{\mathbf{X}}_{i}(k), \\
\mathbf{P}_{i}(k+1 \mid k)= & \boldsymbol{\Phi}(k+1, k) \mathbf{P}_{i}(k) \boldsymbol{\Phi}^{T}(k+1, k) \\
& +\boldsymbol{\Gamma}(k+1, k) \mathbf{Q}_{i}(k) \boldsymbol{\Gamma}^{T}(k+1, k) .
\end{aligned}
$$

(3) Information observation updating:

$$
\begin{aligned}
\mathbf{P}_{i}^{-1}(k \mid k)= & \mathbf{P}_{i}^{-1}(k \mid k-1)+\mathbf{H}_{i}^{T} \mathbf{R}_{i}^{-1}(k) \mathbf{H}_{i}(k), \\
\mathbf{P}_{i}^{-1}(k \mid k) \widehat{\mathbf{X}}_{i}(k \mid k)= & \mathbf{P}_{i}^{-1}(k \mid k-1) \widehat{\mathbf{X}}_{i}(k \mid k-1) \\
& +\mathbf{H}_{i}^{T}(k) \mathbf{R}_{i}^{-1}(k) \mathbf{Z}_{i}(k) .
\end{aligned}
$$

(4) Information fusion:

$$
\begin{aligned}
& \mathbf{P}_{g}=\left[\mathbf{P}_{1}^{-1}+\mathbf{P}_{2}^{-1}+\cdots+\mathbf{P}_{m}^{-1}\right]^{-1}, \\
& \widehat{\mathbf{X}}_{g}=\mathbf{P}_{g} \cdot\left[\mathbf{P}_{1}^{-1} \widehat{\mathbf{X}}_{1}+\mathbf{P}_{2}^{-1} \widehat{\mathbf{X}}_{2}+\cdots+\mathbf{P}_{m}^{-1} \widehat{\mathbf{X}}_{m}\right]^{-1} .
\end{aligned}
$$

\subsection{Objective Function}

2.3.1. Maximum Detection Accuracy. The detection probability is established in the alliance model. After the Kalman filter is used to estimate the state of the target measurement, the joint detection probability density will be $p d_{j}$. The total probability of the detection of the target by the agent $i$ can be defined as follows: whether the $i_{1}$ (except $i_{0}$ ) sensor is added to the dynamic alliance to target under the influence of the complex environment for the detection accuracy $p d_{j}$, the 


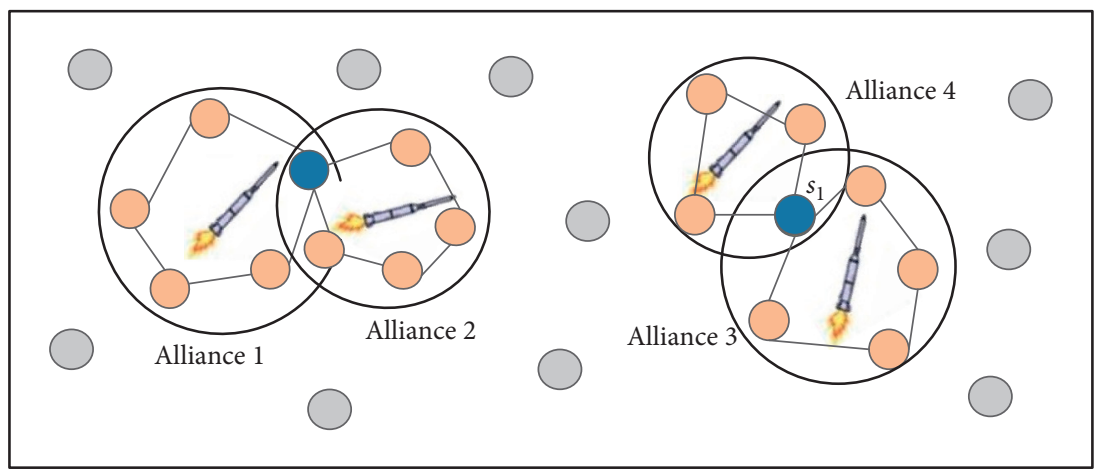

FIGURE 1: Diagram of mixed alliance formation process.

corresponding bracket item should be added or removed every time a sensor is added or removed from the alliance.

$$
p d_{j}=1-\left(1-p d_{i_{0} j}\right)\left(1-p d_{i_{h} i_{1}}{ }^{c u e} p d_{1 j}\right)\left(1-p d_{i_{h} i_{2}}{ }^{c u e} p d_{2 j}\right)\left(1-p d_{i_{h} i_{3}}{ }^{c u e} p d_{3 j}\right) \cdots\left(1-p d_{i_{h} i_{m}}{ }^{c u e} p d_{m j}\right) \text {, }
$$

where $p d_{m j}$ is the detection rate of the $m$ th working agent for the target number $j . p d_{i_{0} i_{m} j}{ }^{c u e}$ is defined as the probability that the No. $n$ agent prompts the No. $m$ agent successfully.
We establish the optimization model of multisensor dynamic alliance for target detection:

$$
\operatorname{Max} P=\sum_{j=1}^{n} p_{j}=\left[1-\exp \left(\ln \left(1-p d_{i_{0} j}\right)^{H_{i_{0} j}}+\sum_{i=1}^{m} \ln \left(1-p d_{i_{n} i_{m}}{ }^{c u e} p d_{i_{0} j}\right)^{H_{i_{0} j}}\right)\right] .
$$

The geometric precision attenuation factor is used as the tracking precision of the sensor [16].

$$
\rho=(\operatorname{Tr}(P(k \mid k)))^{1 / 2} .
$$

2.3.2. Minimum Detection Time. The less the time it takes for $N$ sensor alliances to perform $M$ tasks, indicating that each task is executed by the nearest sensor alliance, which greatly improves the allocation of sensor resources, the better the task accomplished will be. Assuming that the total detection time is $T$, the following objective functions can be obtained:

$$
\operatorname{Min} T=\min \sum_{i=1}^{N} \sum_{j=1}^{M} t_{i j}
$$

2.3.3. Minimum Detection Consumption. The consumption of accomplishing tasks mainly consists of two parts: the consumption of itself and the consumption of sensors. Assuming that the consumption of each sensor is $c_{j}(j=$ $1, \cdots, m)$ and each sensor alliance is $\mathbf{A}_{m \times n}$, where $a_{i j}=1$ means that the sensor tracks the target and $a_{i j}=0$ means that the sensor does not track the target. The following objective functions can be obtained:

$$
\begin{aligned}
\operatorname{Min} C & =\alpha \min \sum_{i=1}^{n} \sum_{j=1}^{m} c_{j} H_{i j}, \\
H_{i_{h} j} & = \begin{cases}1, & \text { the } h-\text { th sensor joins the dynamic alliance for detecting target } j, \\
0, & \text { the } h-\text { th sensor does not join the dynamic alliance for detecting target } j .\end{cases}
\end{aligned}
$$

\subsection{Multisensor Cross-Cueing Algorithm}

2.4.1. Relationship between Multisensor Cross-Cue and Hybrid Dynamic Alliance. The establishment scheme of multisensor hybrid dynamic alliance is based on a certain understanding of the target information. Before the formation of the alliance, it needs to detect the target information. This process is accomplished by multisensor cross- 
cue. After the establishment of the alliance scheme, sensors in the alliance share their own resources and tasks and its essence is also multisensor cross-cutting. The process of alerting is a microadjustment process, which is completed through coordination and negotiation among sensors. This process also belongs to the scope of multisensor cross-alerting. Therefore, multisensor cross-cue occurs from time to time in the process of building multisensor hybrid dynamic alliance, which runs through the whole process of building multisensor alliance. The relationship between multisensor cross-cue and multisensor hybrid dynamic alliance is shown in Figure 2.

\subsubsection{Design of Multisensor Cross-Cueing Algorithm.} From the above analysis, we can see that the problem of solving multisensor hybrid dynamic alliance is essentially a discrete multiobjective assignment problem. The formation and connection process of multisensor hybrid alliance can be realized by establishing multisensor cross-cueing algorithm. At present, the algorithm widely used in this kind of problem is swarm intelligence algorithm represented by particle swarm optimization algorithm. However, in actual multisensor alliance, swarm intelligence algorithm is widely used. In the process of target detection, because the battlefield environment and the target state are changing rapidly, the fitness values corresponding to the individual optimal position and the global optimal position are changing in the dynamic environment, which makes the particles easy to fall into the optimization of the previous environment, and the effect of seeking the optimal solution of the model in the dynamic environment is unsatisfactory.

The particle swarm algorithm is a heuristic algorithm with a fairly simple principle. Compared with other bionic algorithms, it requires fewer codes and parameters. Use the best point of the current search to replace the global optimal solution. To a large extent, this is a single-item information-sharing mechanism and it is less affected by the dimension of the problem. The ant colony algorithm has a slow convergence speed and can easily to fall into a local optimum. The basic ant colony algorithm generally requires a long search time and is prone to stagnation. GA has no memory. The previous knowledge is destroyed as the population changes. Compared with GA, PSO does not require coding and no crossover and mutation operations. The particles are only updated through internal speed, so the principle is simpler, the parameters are fewer, and the implementation is easier. Therefore, a cross-cueing algorithm based on discrete dynamic particle swarm optimization (DPSO) with sensitive particles is proposed to solve this kind of problem.

The sensitive particle-based dynamic discrete particle swarm optimization (DPSO) is a typical dynamic particle swarm optimization (DPSO). It generates sensitive particles randomly when the algorithm is initialized, calculates the fitness of sensitive particles in each iteration, and considers that the environment has changed when the fitness value changes. The response is to reinitialize the particle position and velocity in a certain proportion. The proposed algorithm mainly improves the basic particle swarm optimization algorithm in the following three aspects:
(1) A detection mechanism is introduced to enable the population or particle to acquire the ability to perceive changes in the external environment.

(2) A response mechanism is introduced to update the population to adapt to the dynamic environment after detecting the changes in the environment.

(3) The position of particles in the algorithm represents the hybrid dynamic alliance scheme generated for the target, which can be expressed as a matrix. The matrix is a $0-1$ matrix, where 0 means that the sensor does not join the hybrid dynamic alliance to track the target and 1 means that the sensor joins the hybrid dynamic alliance to track the target.

The dynamic discrete particle swarm optimization (DPSO) algorithm based on sensitive particles is mainly divided into seven parts:

(1) The initialization module initializes the position and velocity of particles in the particle swarm

(2) The sensitive particle initialization module initializes the position of sensitive particles

(3) The fitness module calculates the fitness of particles in the current environment

(4) The particle evolution module updates the particle velocity and position according to the current individual optimal particle and population optimal particle

(5) The alliance is formed and connected according to the updated particle velocity and position

(6) The sensitive particle fitness calculation module calculates sensitive particle fitness value according to the current environment

(7) The population reinitialization module refers to the reinitialization of particle position and velocity in a certain proportion when the fitness value of sensitive particles exceeds the threshold value

The particle velocity update equation is as follows:

$V_{k+1}=\omega V_{k}+c_{1} r_{1}\left(P_{b}-X_{k}\right)+c_{2} r_{2}\left(P_{g}-X_{k}\right)$.

The update equation of particle position is as follows:

$$
X_{k+1}=X_{k}+V_{k+1} \text {. }
$$

Here, $\omega$ is the inertia weight, $k$ is the current iteration number, $V_{k}$ is the current particle velocity, $c_{1}$ and $c_{2}$ are the acceleration factors, and $r_{1}$ and $r_{2}$ are the random numbers distributed between them. In order to prevent blindly searching for particles, their positions and velocities are limited to certain intervals $\left[-X_{\max }, X_{\max }\right]$ and $\left[-V_{\max }, V_{\max }\right]$.

The fitness equations of particles and sensitive particles are as follows:

$$
\text { fitness }(i)=\operatorname{position} x_{i}+\text { position } y_{i}
$$

Among them, fitness $(i)$ is the fitness value of particle and position $x_{i}$ and position $y_{i}$ are the positions of particle $i$. 


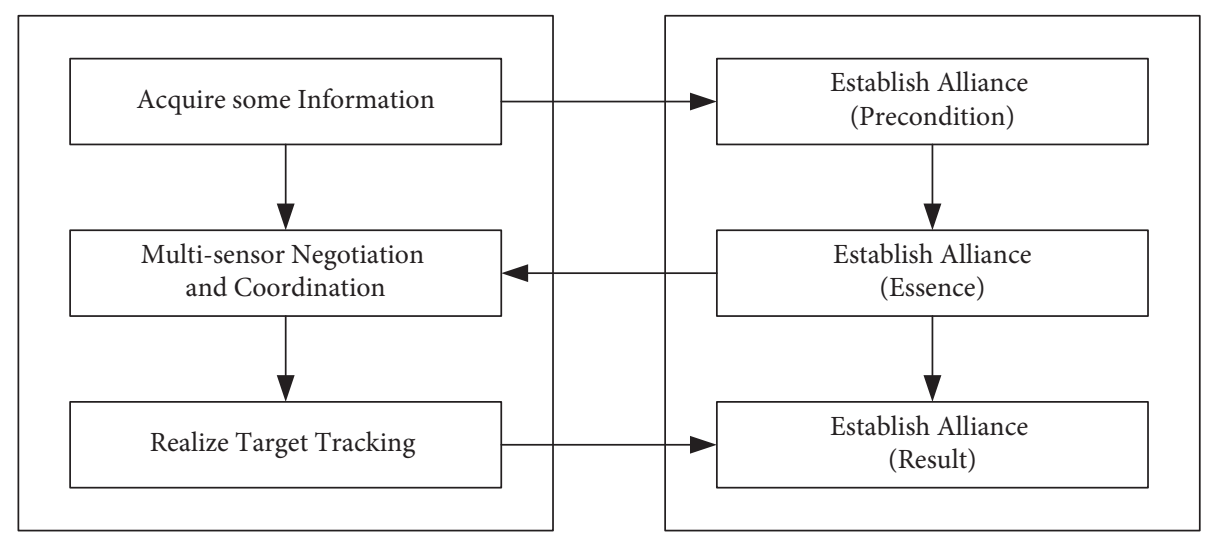

Figure 2: Relationship between multisensor cross-cue and mixed dynamic alliance.

Equation (17) shows that the higher the corresponding height of the particle, the better the fitness of the particle.

The algorithm flow is shown in Figure 3.

\subsubsection{Predict and Re-Predict Handover Mechanism of Hybrid} Multisensor Alliance. After the establishment of hybrid dynamic alliance, because of the large coverage of early warning and detection network composed of multisensors, each sensor cannot respond to the operational requirements in time. At the same time, the estimation time of the target state is changing. It is in a dynamic environment. When the actual detection accuracy of the target at a certain $k$ time does not reach the prediction accuracy, it is very easy to cause the following loss in the process of alliance handover. In order to reduce the probability of alliance losing the target, this paper proposes a mechanism of "predict and repredict" to better realize alliance handover. The process of sensor alliance formation and handover is shown in Figure 4.

The specific implementation steps are as follows:

(1) Selecting the members of the alliance and forming the alliance.

(2) Observing the target, and the observed value is $\mathbf{Z}_{1}(k), \mathbf{Z}_{2}(k), \ldots, \mathbf{Z}_{m}(k)$.

(3) Predicting the target information of the time $k$ according to the target information of the time $k+1$ obtained by the FKF algorithm.

(4) In time $k+1$, since the sensor and target state estimation are in dynamic change, when the target detection cannot be achieved within the prediction range, the prediction will fail. The dichotomy method should be used to reduce the sampling period of time and predict again until the detection accuracy is accurate enough to meet the conditions.

\section{Simulations}

3.1. Parameter Settings. The sampling interval is $1 \mathrm{~s}$, the sampling times are 300, 200 Monte Carlo experiments are carried out, and the simulation time is set to $1000 \mathrm{~s}$.

Table 1 gives the location information of three targets, and Table 2 gives the performance indicators of

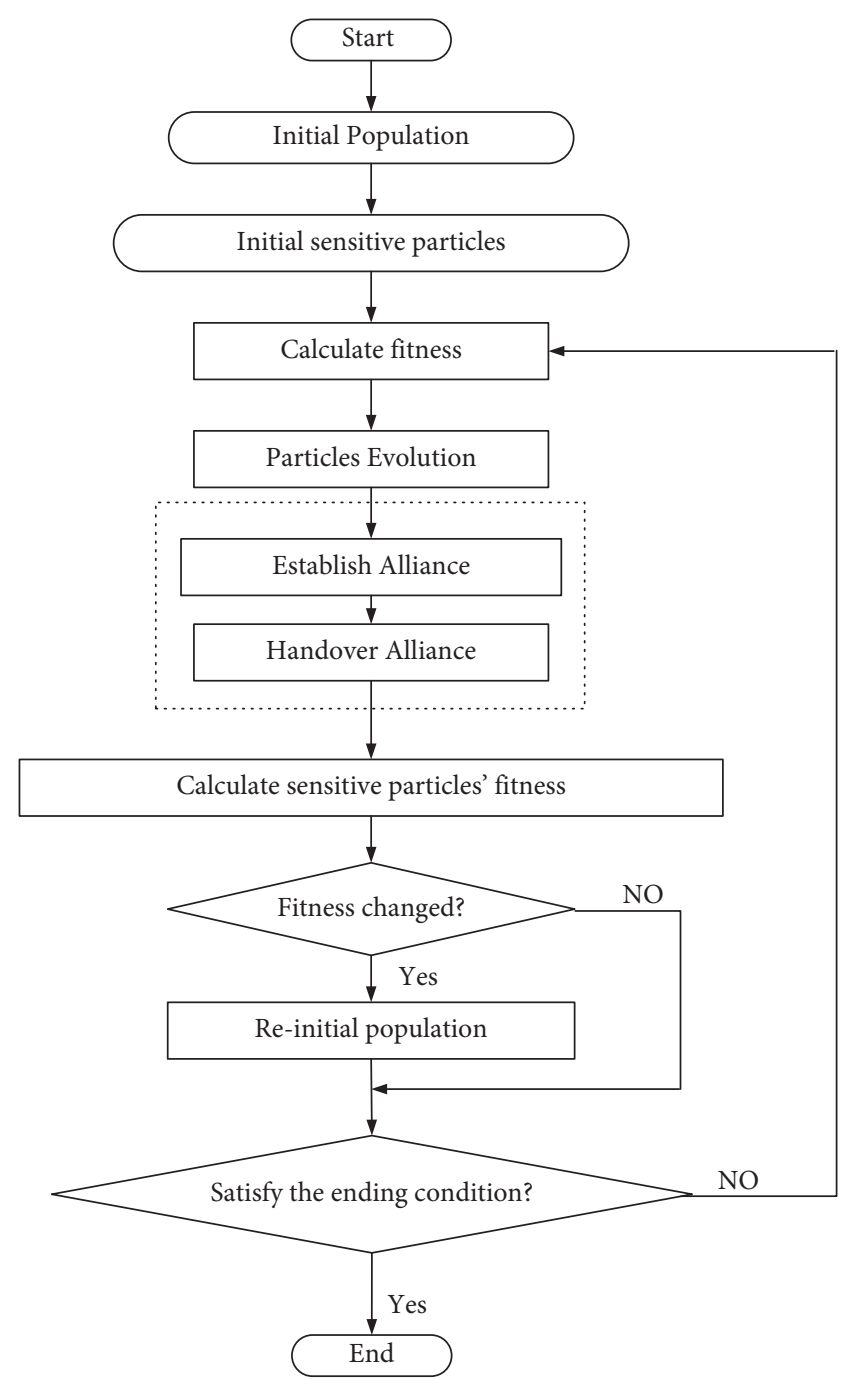

FIgURE 3: Flow chart of the algorithm.

each sensor deployed in the sensor network around the target.

Based on the information shown in Tables 1 and 2, the target trajectory and sensor deployment diagram in Figure 5 can be obtained. 


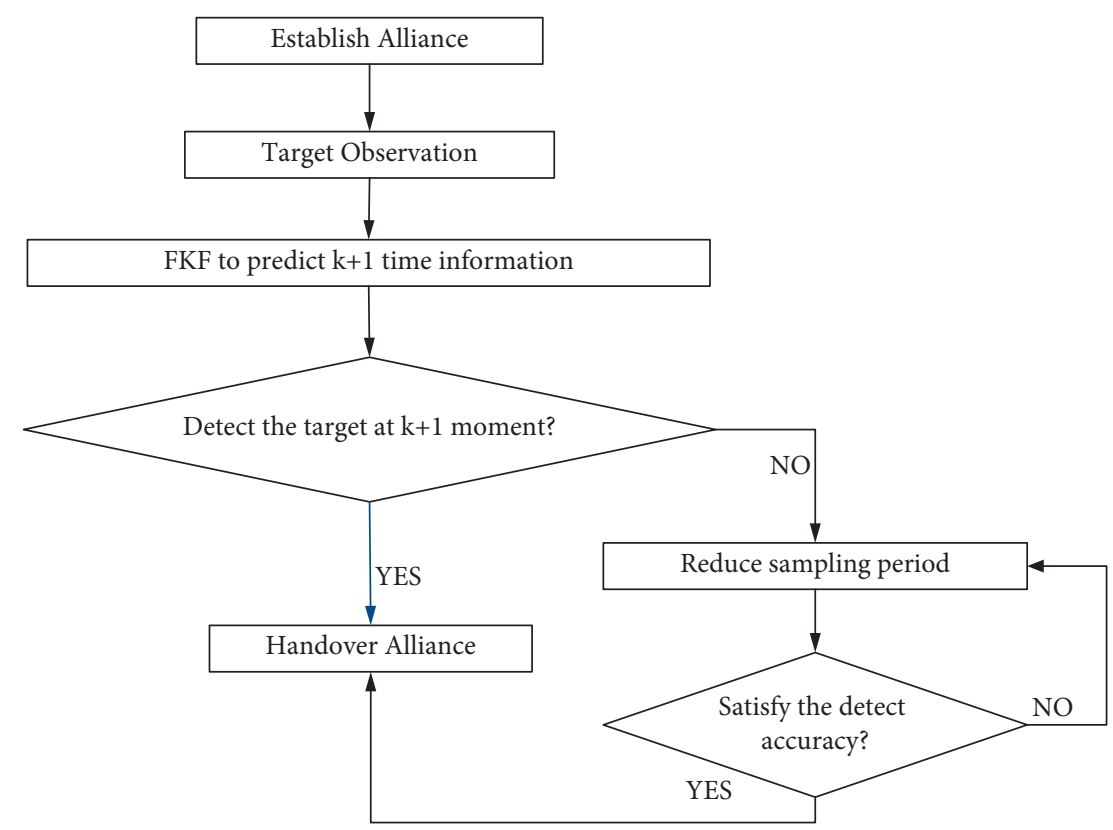

FIgURE 4: Alliance formation and transition process.

TABLE 1: Target information.

\begin{tabular}{lccc}
\hline & Target 1 & Target 2 & Target 3 \\
\hline Initial velocity $(\mathrm{m} / \mathrm{s})$ & 200 & 200 & 200 \\
Initial longitude $\left(^{\circ}\right)$ & 0.5977 & 0.6107 & 0.725 \\
Initial latitude $\left({ }^{\circ}\right)$ & 1.900 & 1.8958 & 1.910 \\
Initial altitude $(\mathrm{m})$ & 200 & 10000 & 5000 \\
\hline
\end{tabular}

TABle 2: Sensor ability information.

\begin{tabular}{lccccc}
\hline Sensor & $\left(\right.$ Longitude $\left({ }^{\circ}\right)$, latitude $\left.\left(^{\circ}\right)\right)$ & Cost & Altitude $(\mathrm{m})$ & Detection radius $(\mathrm{m})$ & RMSE in $X, Y$, and $Z(\mathrm{~m})$ \\
\hline 1 & $(108.8,34.50)$ & 0.12 & 3000 & 30000 & 1 \\
2 & $(108.9,35.20)$ & 0.24 & 15000 & 35000 & 1.2 \\
3 & $(108.7,35.75)$ & 0.23 & 17000 & 55000 & 1 \\
4 & $(108.4,35.75)$ & 0.22 & 14500 & 40000 & 0.8 \\
5 & $(108.2,35.60)$ & 0.16 & 22500 & 45000 & 0.9 \\
6 & $(108.0,35.75)$ & 0.18 & 15000 & 50000 & 1.3 \\
7 & $(107.9,35.25)$ & 0.21 & 10000 & 40000 & 1 \\
8 & $(107.8,34.75)$ & 0.09 & 1000 & 45000 & 1.1 \\
\hline
\end{tabular}

The initial parameters of the FKF algorithm are set as follows.

The covariance matrix of observation noise is

$$
\mathbf{R}_{k}=\left[\begin{array}{ccc}
R_{x^{2}} & 0 & 0 \\
0 & R_{y^{2}} & 0 \\
0 & 0 & R_{z^{2}}
\end{array}\right] .
$$

$$
\mathbf{Q}_{k}=0.001 \cdot\left[\begin{array}{cccccc}
T^{5} / 20 & T^{4} / 8 & T^{3} / 6 & 0 & 0 & 0 \\
T^{4} / 8 & T^{3} / 6 & T^{2} / 2 & 0 & 0 & 0 \\
T^{3} / 6 & T^{2} / 2 & T & 0 & 0 & 0 \\
0 & 0 & 0 & T^{5} / 20 & T^{4} / 8 & T^{3} / 6 \\
0 & 0 & 0 & T^{4} / 8 & T^{3} / 6 & T^{2} / 2 \\
0 & 0 & 0 & T^{3} / 6 & T^{2} / 2 & T
\end{array}\right] .
$$

The covariance matrix of system noise is 


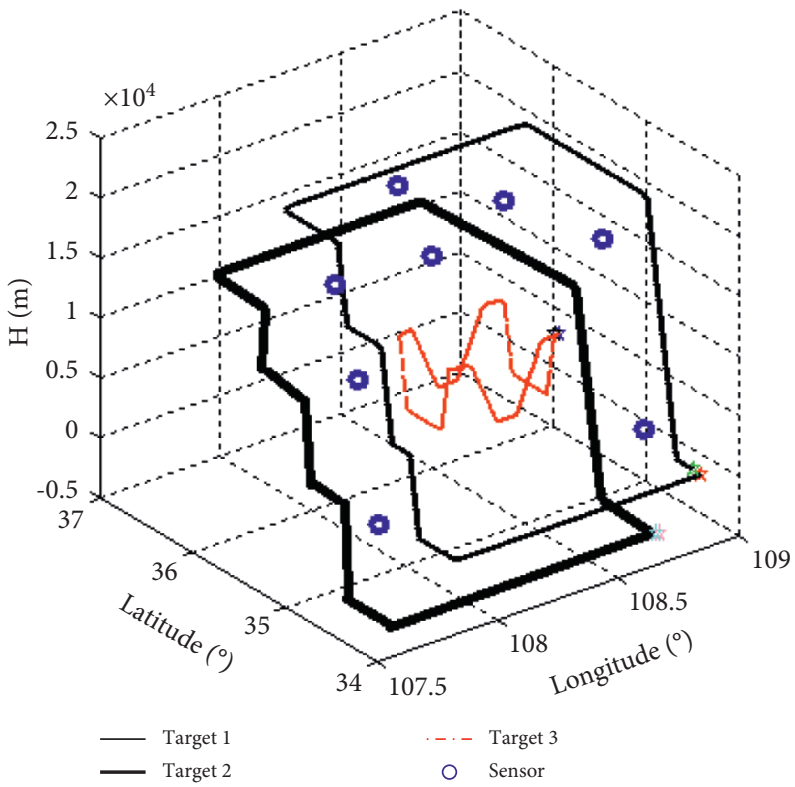

FIGURE 5: Figure of the trajectory of the target and deployment of sensors.

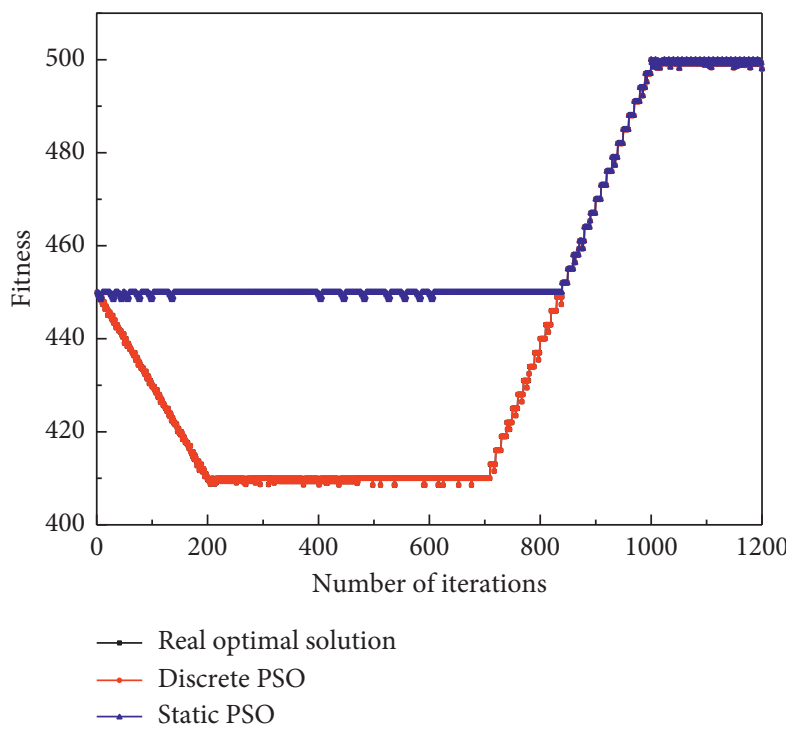

(a)

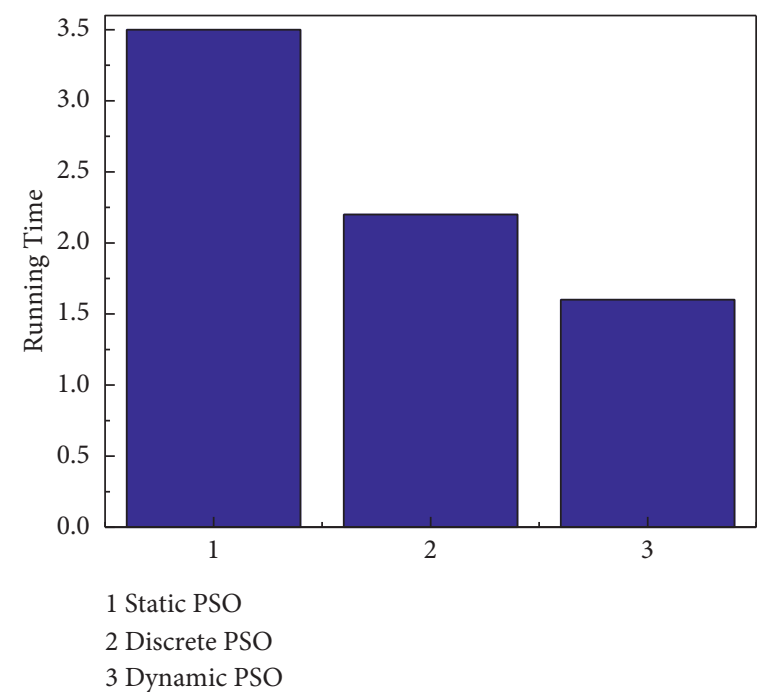

(b)

Figure 6: Continued. 


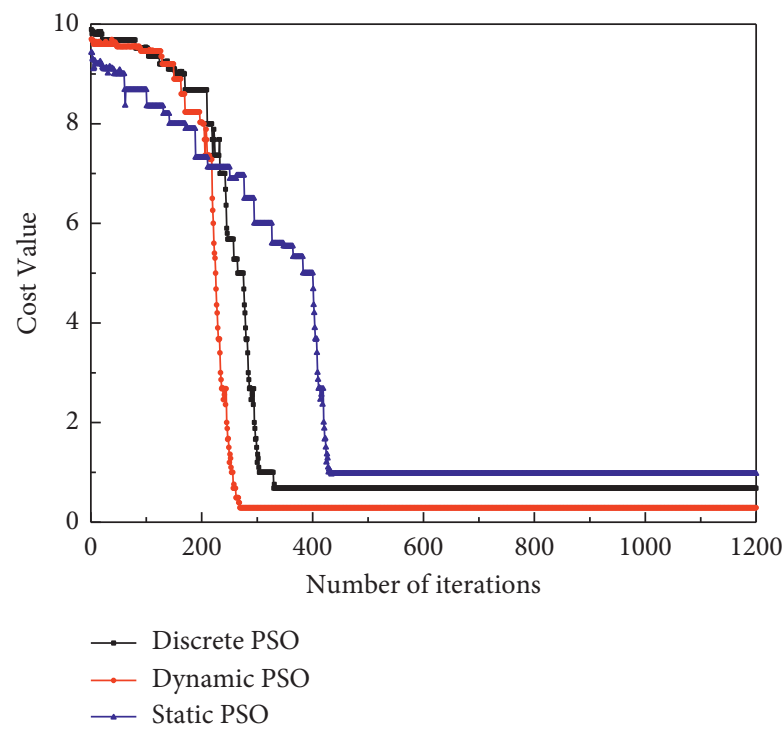

(c)

Figure 6: Performance analysis of the algorithms. (a) Convergence analysis of the algorithms. (b) Running time of the algorithms. (c) Cost value of the algorithms.

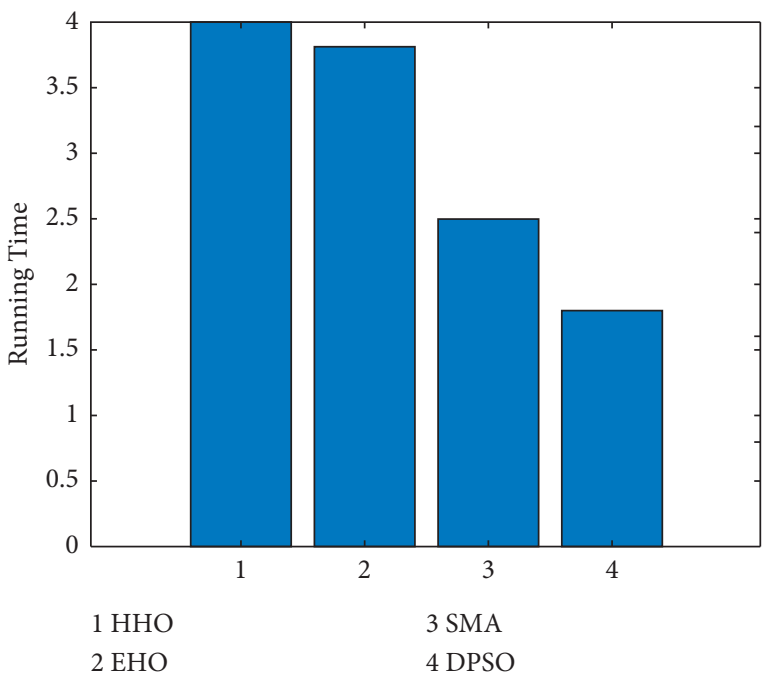

(a)

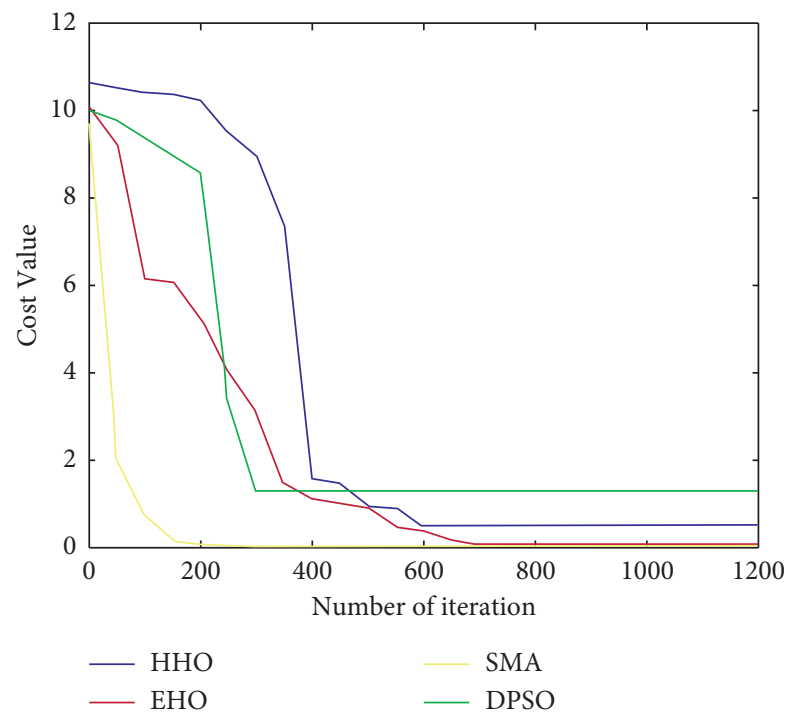

(b)

FIGURE 7: Swarm intelligence algorithm model comparison. (a) Running time of the algorithms. (b) Cost value of the algorithms.

\subsection{Analysis of the Simulation Results}

3.2.1. Performance Simulation Analysis of the Algorithm. The population has 20 particles, 100 evolutionary times, 20 sensitive particles, and 1200 iterations. The dynamic and static particle swarm optimization algorithms are compared and simulated. The results are shown in Figure 6.

Figure 6 shows that in the process of dynamic environment change, both ordinary particle swarm optimization and dynamic particle swarm optimization converge, but ordinary particle swarm optimization shows difficulty to dynamically track the global environment change because of trapping local optimum. Figure 7 shows that the convergence time of the $\mathrm{HHO}$ [37] algorithm is too long under the dynamic model, the EHO [38] algorithm falls into the local optimum prematurely, and the SMA [39] algorithm has higher convergence speed and convergence accuracy but falls into the local optimum solution earlier. However, the dynamic particle swarm optimization (DPSO) algorithm can detect and adapt to the changing dynamic environment. It can solve quickly and consume less energy, so it can track the dynamic global optimum in real time. 

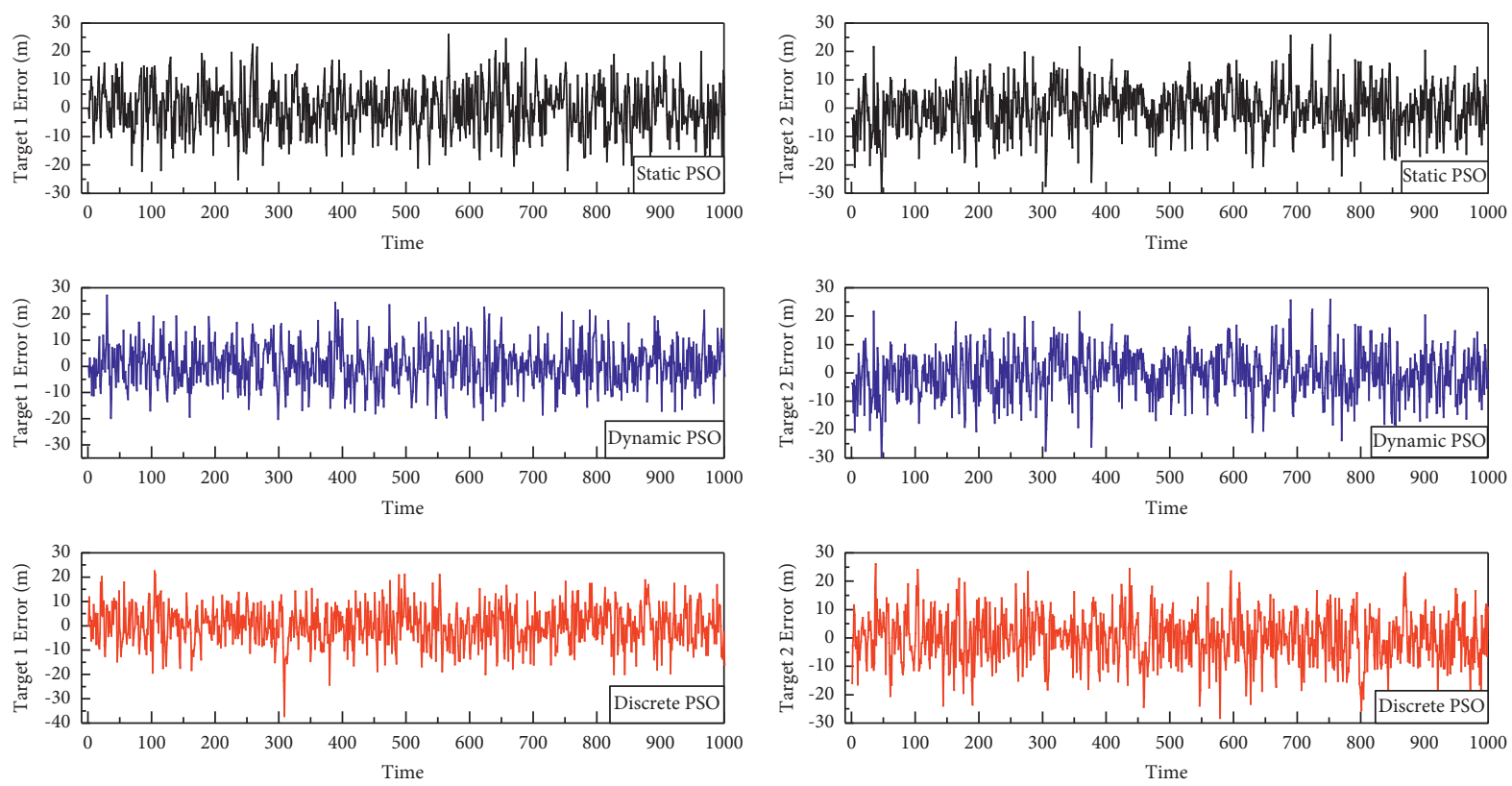

(a)

(b)
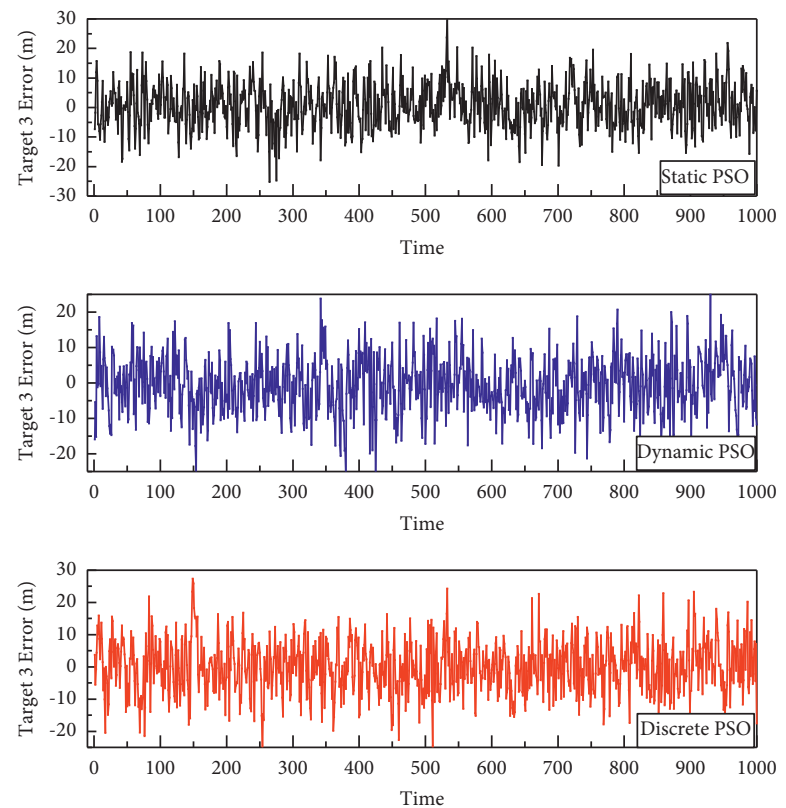

(c)

FIgURE 8: Target tracking error. (a) Target 1 tracking error. (b) Target 2 tracking error. (c) Target 3 tracking error.

3.2.2. Simulation Analysis of the Target Detection Error. The static particle swarm optimization (PSO), the discrete particle swarm optimization (DPSO), and the proposed algorithm are compared, and the detection errors of the three targets are obtained as shown in Figure 8.

From Figure 8, it can be seen that all three particle swarm optimization algorithms can achieve target detection error. Compared with dynamic particle swarm optimization, the dynamic particle swarm optimization algorithm has a smaller detection error range, higher detection accuracy, and better detection of targets. Adapt to the dynamic changes of the combat environment, save time, and improve efficiency.

\subsubsection{Simulation and Analysis of Sensor Hybrid Dynamic} Alliance Formation Process

(1) Performance Comparison of Alliance Formation Methods. In order to illustrate the effectiveness of cross-cueing alliance formation algorithm, simulation experiments are carried out from the perspective of whether cross-cue is used or not.

Figures 8-10 show the detection error of the target and the real-time update schematic of the sensor with or without cross-cue, respectively. The simulation time is set to $3000 \mathrm{~s}$.

The simulation results show that multisensor cross-cue can reduce target detection error, and more sensors will 

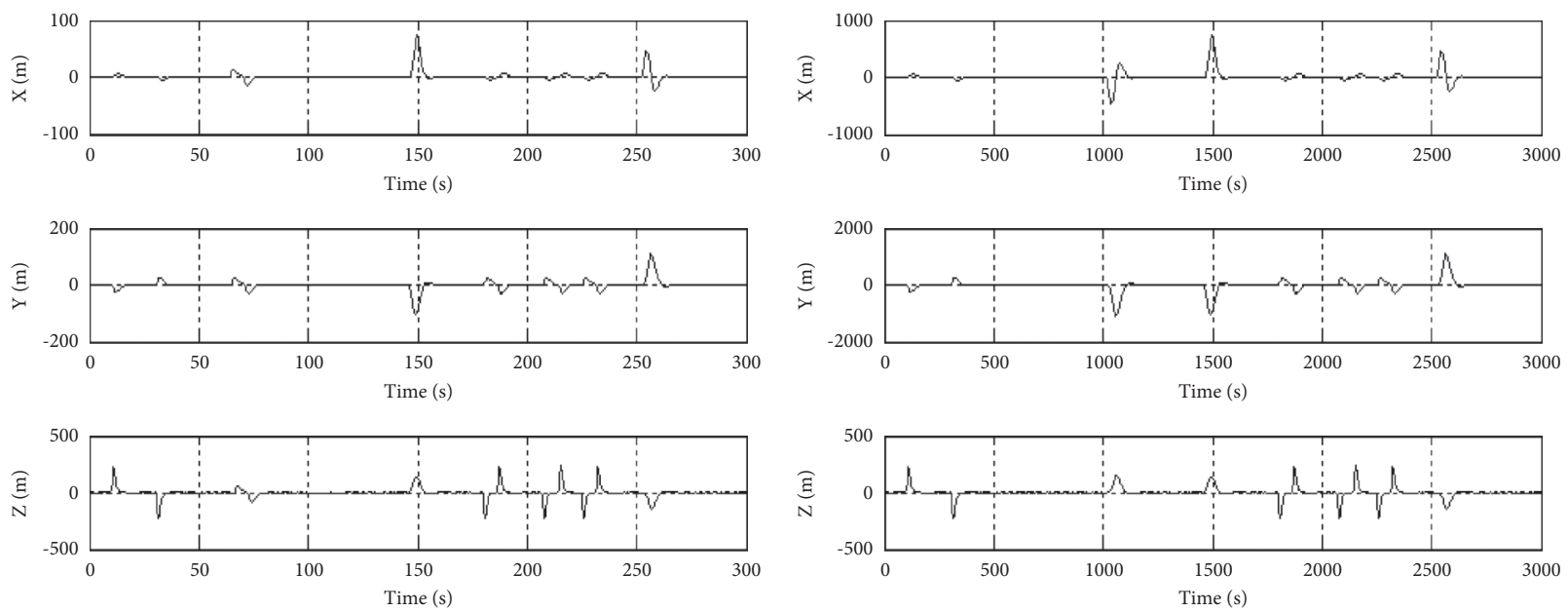

(a)

(b)
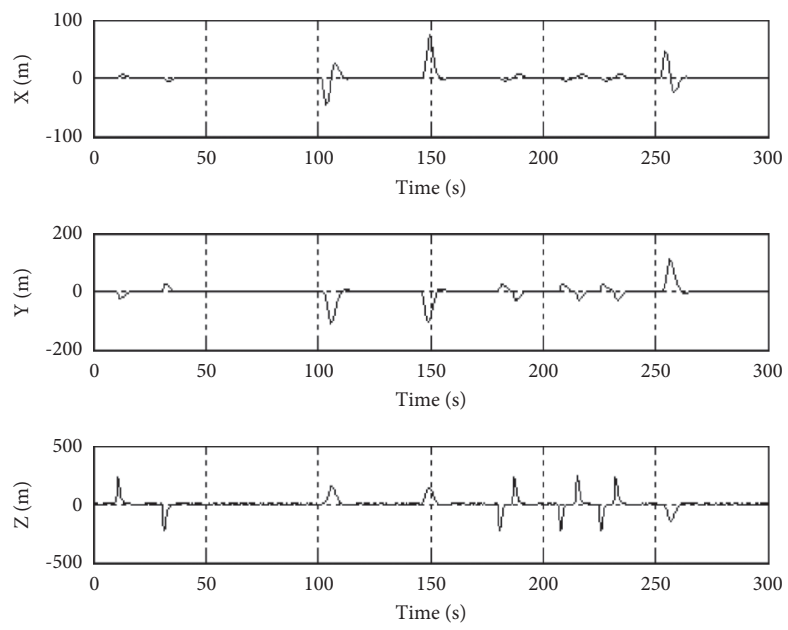

(c)

Figure 9: Target detection error without cross-cue. (a) Target 1 tracking error. (b) Target 2 tracking error. (c) Target 3 tracking error.
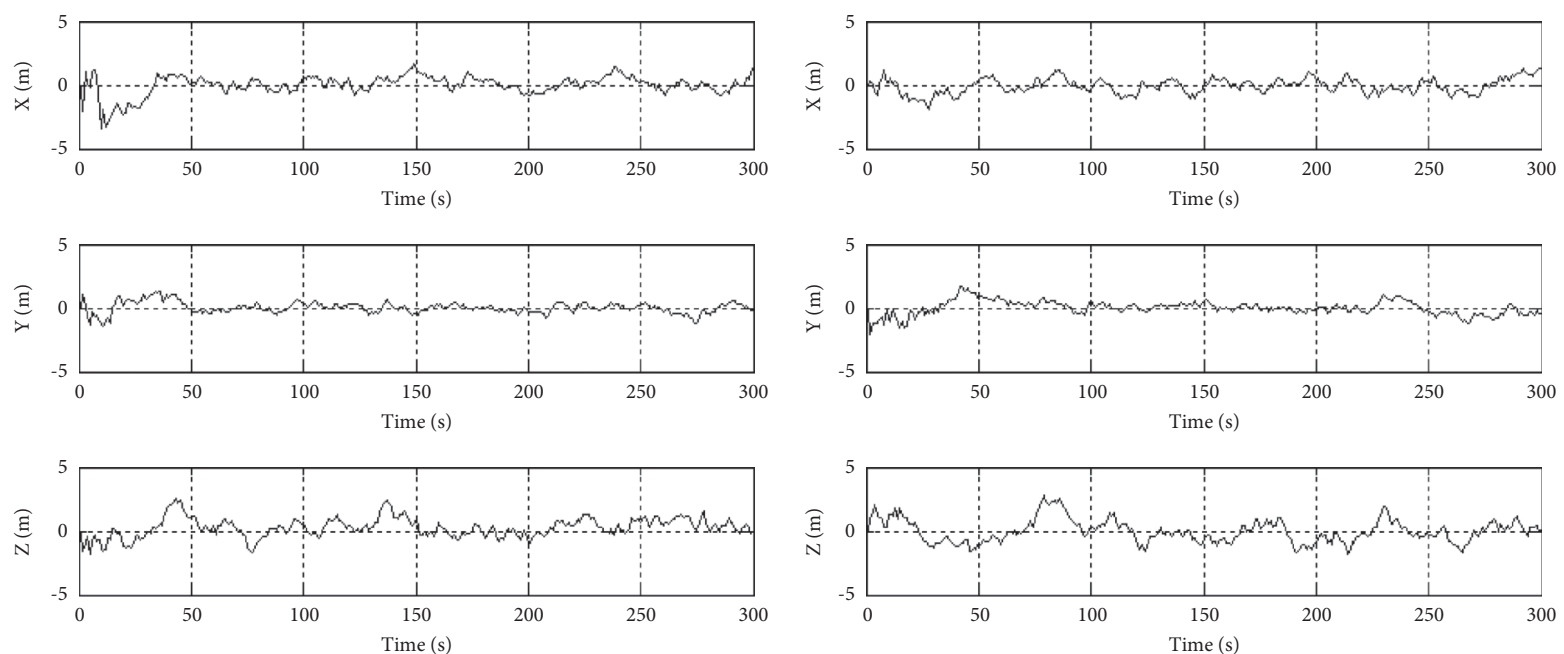

(a)

(b)

Figure 10: Continued. 

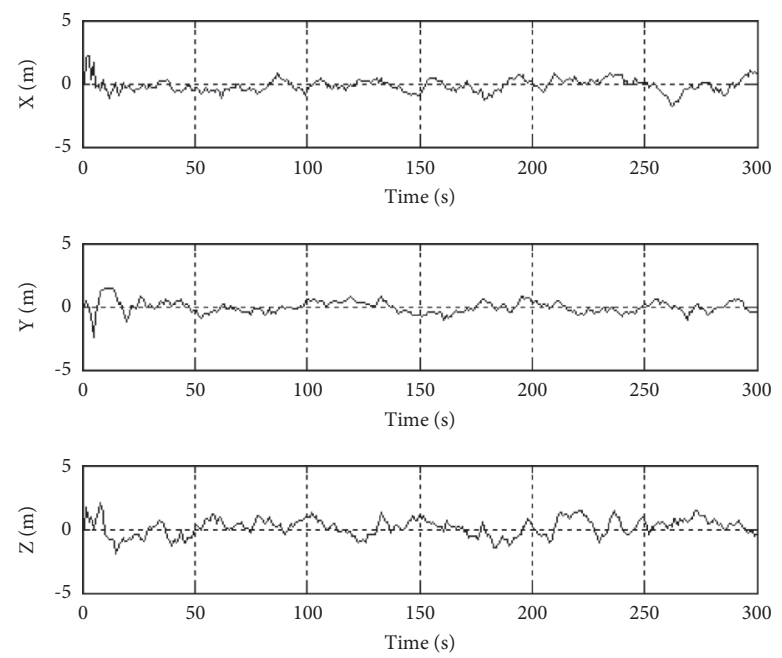

(c)

Figure 10: Target tracking error with cross-cue. (a) Target 1 tracking error. (b) Target 2 tracking error. (c) Target 3 tracking error.

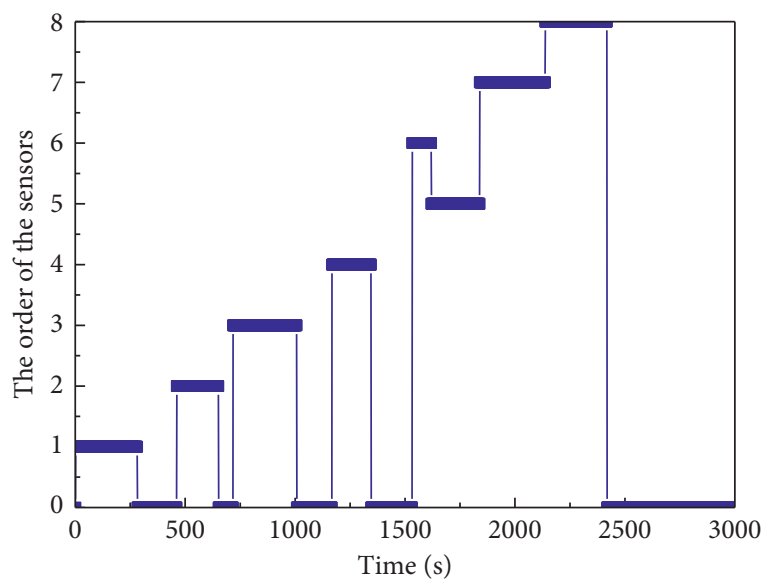

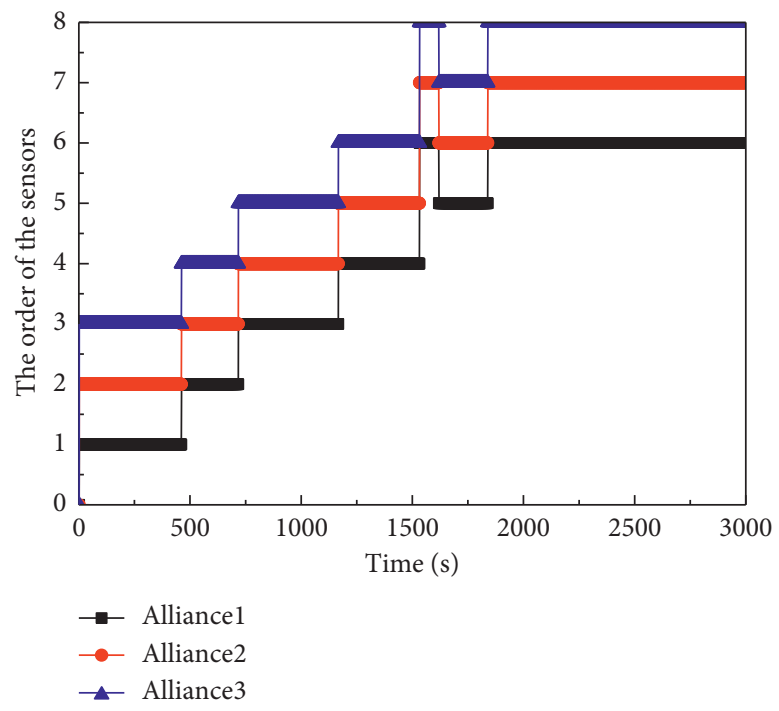

(b)

FIgURE 11: Real-time update diagram of sensors. (a) The sensor response order without cross-cue. (b) The sensor response order with cross-cue.

respond to the requirement of building multisensor hybrid dynamic alliance. The simulation results further show that multisensor cross-cue occurs from time to time in the process of building multisensor hybrid dynamic alliance, which runs through the whole process of building multisensor alliance.

(2) Performance Comparison of Alliance Handover Methods. In order to illustrate the validity of the "predictive re-prediction" mechanism in the handover process of sensor alliance, the proposed mechanism is compared with the two handover mechanisms of "measurement re-update" and "prediction is update." The simulation time is set to 300 seconds. The simulation results are shown in Figure 11.

Figure 12 shows the comparison of detection errors for three targets with different handover mechanisms. The proposed handover mechanism has the smallest error and better convergence than the other two handover mechanisms. This is because the hybrid alliance formed by sensors in the dynamic environment can provide accurate measurement values, thus greatly reducing the detection error. 


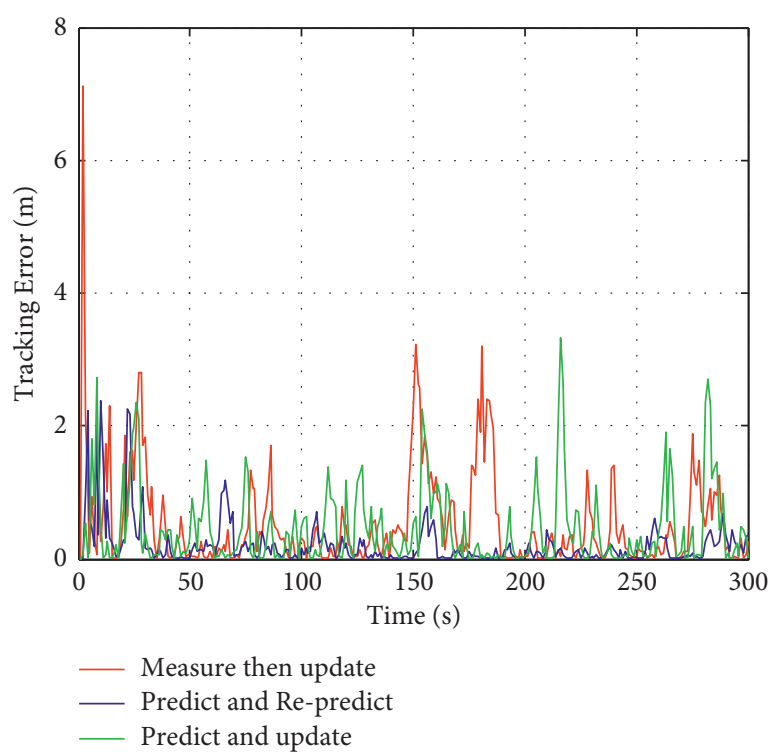

(a)

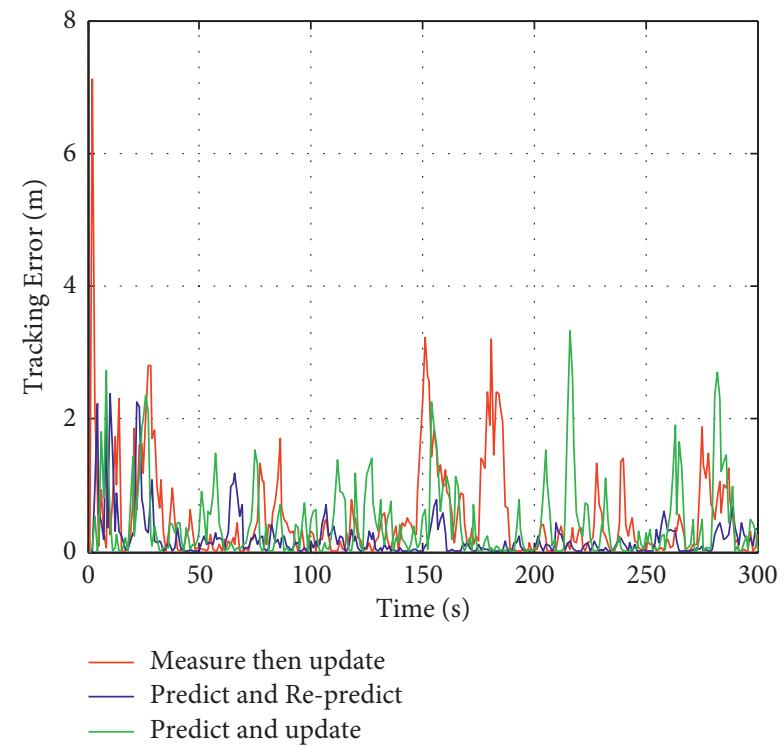

(b)

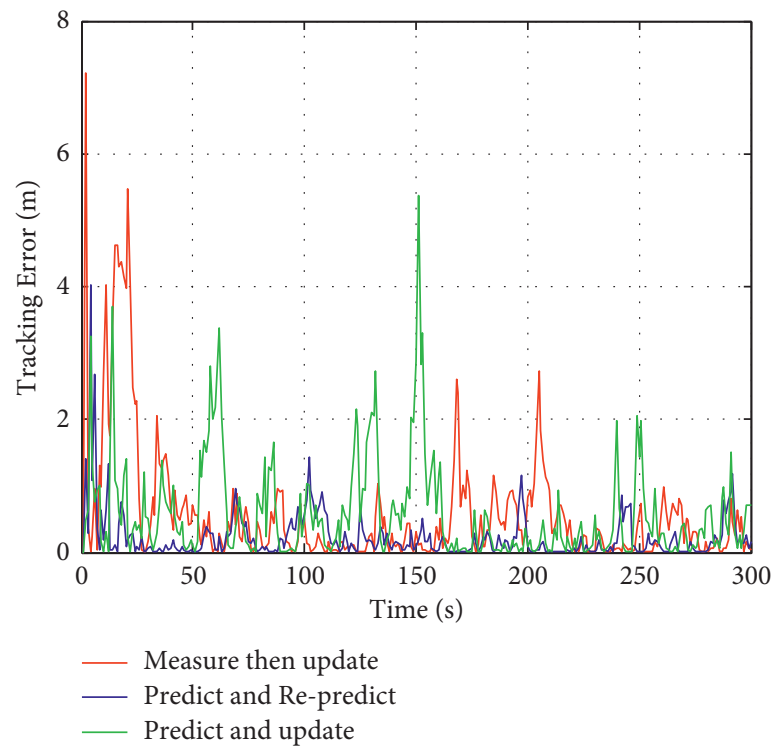

(c)

Figure 12: Target tracking error using different methods. (a) Target 1 tracking error. (b) Target 2 tracking error. (c) Target 3 tracking error.

\section{Conclusions and Future Work}

This paper mainly focuses on the application of multisensor cross-cueing technology to solve the formation and handover of multisensor hybrid dynamic alliance.

(1) Firstly, the model of multisensor hybrid dynamic alliance is proposed. Secondly, the relationship between multisensor cross-cue and multisensor hybrid dynamic alliance is analyzed, which shows that multisensor cross-cue runs through the whole process of the formation and update of dynamic alliance.

(2) A cross-cueing algorithm based on sensitive particles and a hybrid alliance mechanism of "predict and repredict" are proposed. Finally, the simulation verification is carried out.
(3) The multisensor hybrid dynamic alliance proposed in this paper meets the actual needs of the dynamic change of the target state and battlefield environment and completes the target detection task while realizing the formation and handover of the alliance.

(4) Compared with other similar swarm intelligence algorithms (HHO, EHO, SWA), the algorithm proposed in this paper has better solution quality and convergence speed and the effectiveness of the algorithm is verified in experiments.

(5) Compared with other handover mechanisms, the predictive re-prediction mechanism proposed in this paper is more suitable for the dynamic battlefield environment. 
In future work, we will further study the application objects and applicable scenarios of the sensing technology in sensor work, such as the UAV cluster target that everyone is very concerned about or the application of ultrahigh-speed target scenarios, combined with swarm intelligence algorithms such as $\mathrm{HHO}, \mathrm{MFO}$, and other algorithms for targeted improvements to improve model performance.

\section{Data Availability}

The data used to support the findings of this study have been deposited in [16-20] in the paper.

\section{Conflicts of Interest}

The authors declare that they have no conflicts of interest.

\section{Acknowledgments}

This research was funded by the National Natural Science Foundation of China, under grant no. 61603410, Natural Science Basic Research in Shaanxi Province of China, under grant no. 2018JQ6024, and Young Talent Fund of University Association for Science and Technology in Shaanxi, China, under grant no. 20170107.

\section{References}

[1] S. T. Qu and H. H. Yang, "Multi-target detection and detecting of video sequence based on Kalman_BP neural network," Infrared and Laser Engineering, vol. 42, no. 9, pp. 2553-2560, 2013.

[2] H. B. Luo, L. Y. Xu, B. Hu, and Z. Chang, "Status and prospect of target detecting based on deep learning," Infrared and Laser Engineering, vol. 46, no. 5, pp. 1-6, 2017.

[3] C. F. Liu, G. Wang, Z. M. Zhang, F. Chen, Y. Liu, and X. N. Mei, "Technical requirements analysis of air defense system intercepting fast attack weapons in near space," Modern Defense Technology, vol. 43, no. 5, pp. 18-25, 2015.

[4] Z.-H. Li, C. Y. Liu, P. Ni, J. Yu, and L. I. Song, "Review on multi-sensor cooperative mission planning in anti-TBM system," Journal of Astronautics, vol. 37, no. 1, pp. 29-38, 2016.

[5] Q. Liu, Study on Cooperative Network Collaborative Detecting Algorithm with Multi-Sensor, Xidian University, Xi'an, China, 2013.

[6] Q. Fu, G. Wang, X. K. Guo, C. Y. Liu, and X. K. Zhang, "Requirements analysis on collaborative detection and detecting of near space high-speed targets," Systems Engineering and Electronics, vol. 37, no. 4, pp. 757-762, 2015.

[7] G. Zolotnik and J. S. Rosenshein, "One, two, many: alliances in multi-agent systems," in Proceedings of the Fifth European Workshop on Modelling Autonomous Agents in a Multi-Agent World (MAAMAW93), LNAI, vol. 957, Springer-Verlag, Neuchatel, Switzerland, August 1993.

[8] O. Shehory and S. Kaus, "Alliance formation among autonomous agents: strategies and complexity," in Proceedings of the Fifth European Workshop on Modeling Autonomous Agents in a Multi-Agent World (MAAMAW93), LNAI, vol. 957, SpringerVerlag, Neuchatel, Switzerland, August 1993.

[9] M. Liu, H. H. Li, and Y. Shen, "Research on task allocation technique for aerial target detecting based on wireless sensor network," Journal of Astronautics, vol. 28, no. 4, pp. 960-961, 2007.

[10] J. X. Chen and H. B. Yu, "An updating scheme of the working dynamic alliance for collaborative task allocation in wireless sensor networks," Chinese Journal of Sensors and Actuators, vol. 22, no. 4, pp. 499-504, 2009.

[11] G. L. Chen, W. Z. Guo, and Y. Z. Chen, "Research on dynamic alliance of task allocation and its algorithm in wireless sensor network," Journal of Communication, vol. 30, no. 11, pp. 48-55, 2009.

[12] Z. Zheng and W. Q. Xiong, "Sensor adaptive task scheduling algorithm based on multi-agent system theory and dynamic alliance," Computer Engineering, vol. 41, no. 12, pp. 58-63, 2015.

[13] X. M. Fu, M. W. Zhao, and Y. Li, "Dynamic alliance formation among UCAVs communication networks," Systems Engineering and Electronics, vol. 39, no. 1, pp. 193-197, 2017.

[14] G. Shi, F. Wu, L. Zhang, S. Zhang, and C. Guo, "An airborne multi-sensor task allocation method based on improved particle swarm optimization algorithm," Xibei Gongye Daxue Xuebao/Journal of Northwestern Polytechnical University, vol. 36, no. 4, pp. 722-727, 2018.

[15] X. H. Diao, Y. W. Fang, S. B. Xiao, and D. H. Mao, “Task allocation in cooperative air combat based on multi-agent alliance," Journal of Beijing University of Aeronautics and Astronautics (Social Sciences Edition), vol. 40, pp. 1268-1275, 2014.

[16] C. Pang, G. L. Shan, X. S. Duan, and G. G. Xu, "A multi-mode sensor management approach in the missions of target detecting and detecting," Electronics, vol. 8, no. 71, pp. 1-18, 2019.

[17] G. G. Xu, C. Pang, X. S. Duan, and G. L. Shan, "Multi-sensor optimization scheduling for target detecting based on PCRLB and a novel intercept probability factor," Electronics, vol. 8, no. 140, pp. 1-22, 2019.

[18] G. W. Ng, K. H. Ng, and L. T. Wong, "Sensor managementcontrol and cue," in Proceedings of the 3rd International Conference on Information Fusion, pp. 100-113, Singapore, July 2000.

[19] T. Yan and C. Z. Han, "Sensor management for multi-target detection and detecting based on PCRLB," in Proceedings of the 2017 20th International Conference on Information Fusion, pp. 10-13, Xi'an, China, July 2017.

[20] C. Shi, F. Wang, M. Sellathurai, and J. Zhou, "LPI optimization framework for target detecting in radar network architectures using information theoretic criteria," International Journal of Antennas and Propagation, vol. 2014, Article ID 654561, 10 pages, 2014.

[21] J. Y. Sang, P. S. Anish, M. Seo, C. H. Han, P. Minho, and K. E. Lee, "Joint spectrum sensing and resource allocation optimization using genetic algorithm for frequency hoppingbased cognitive radio networks," International Journal of Communication Systems, vol. 31, Article ID e3733, 2018.

[22] X. T. Yang, J. F. Feng, and Y. Feng, "A multi sensor-target assignment algorithm based on genetic particle swarm optimization," Electronics Optics and Control, vol. 18, pp. 5-8, 2011.

[23] C.-F. Wang and K. Liu, "A novel particle swarm optimization algorithm for global optimization," Computational Intelligence and Neuroscience, vol. 2016, Article ID 9482073, 9 pages, 2016.

[24] S. Chen, Y. Liu, L. Wei, and B. Guan, "PS-FW: a hybrid algorithm based on particle swarm and fireworks for global 
optimization," Computational Intelligence and Neuroscience, vol. 2018, Article ID 6094685, 27 pages, 2018.

[25] A. G. Fei, L. Y. Zhang, G. Liu, and Y. Wang, "The technique for air-to-air missile guidance superiority handover based on particle swarm hybrid algorithm," Journal of Astronautics, vol. 34, no. 3, pp. 340-346, 2013.

[26] H. Bao, B. Zhang, C. Li, and Z. Yao, "Mobile anchor assisted particle swarm optimization (PSO) based localization algorithms for wireless sensor networks," Wireless Communications and Mobile Computing, vol. 12, no. 15, pp. 1313-1325, 2012.

[27] Y. Zhou, R. Zhao, Q. Luo, and C. Wen, "Sensor deployment scheme based on social spider optimization algorithm for wireless sensor networks," Neural Processing Letters, vol. 48, no. 1, pp. 71-94, 2018.

[28] S. G. Ying, F. C. Sun, L. H. Hu, H. P. Liu, and X. J. Zhang, "Multi-objective dynamic programming algorithm for aircraft arrival sequencing and runway scheduling," Control Theory \& Applications, vol. 27, no. 7, pp. 827-835, 2010.

[29] L. Zhong, Q. Huang, F. Wu, and G. Chen, "Trade: a truthful online combinatorial auction for spectrum allocation in cognitive radio networks," Wireless Communications and Mobile Computing, vol. 15, no. 9, pp. 1320-1330, 2015.

[30] W. Z. Guo, J.-S. Su, C. Y. Chen, G. L. Chen, and S. O. Computer, "Self-adapted task allocation algorithm with complicated alliance in wireless sensor network," Journal of Communication, vol. 35, no. 3, pp. 1-10, 2014.

[31] A. Carlisle and G. Dozier, "Adapting particle swarm optimization to dynamic environments," in Proceedings of the International Conference on Artificial Intelligence (ICAI 2000), pp. 429-434, Las Vegas, NV, USA, 2000.

[32] Y. Chen, W. Z. Guo, G. L. Chen, and Z. H. Liu, "Dynamic task allocation algorithm with parallel alliance in wireless sensor networks," Journal of Chinese Computer Systems, vol. 3, no. 3, pp. 480-485, 2012.

[33] H. Fan, S. C. Huang, M. F. Gao, and D. Z. Wei, "Research on technique of multi-target detection using multi-sensor crosscueing based on dynamic alliance," Journal of Astronautics, vol. 32, no. 11, pp. 2380-2386, 2011.

[34] C. Pang, S. C. Huang, J. C. Liu, W. Zhao, and D. Z. Wei, "Application of multi-sensor cross cuing technology in sensor alliance," Journal of Xi'an Jiaotong University, vol. 51, no. 7, pp. 148-155, 2017.

[35] M. L. Fu, H. Wang, B. F. Fang, and X. L. Huang, "Task allocation for distributed Self-interested agents," Pattern Recognition and Artificial Intelligence, vol. 31, no. 12, pp. 1061-1073, 2018.

[36] J. Xie, S. Huang, and D. Wei, "Uncertain hybrid multi-sensor alliance dynamic control problem using an uncertain ideal point approach under the PEV principle," IEEE Access, vol. 8, pp. 169385-169395, 2020.

[37] A. A. Heidari, S. Mirjalili, H. Faris, I. Aljarah, M. Mafarja, and H. Chen, "Harris hawks optimization: algorithm and applications," Future Generation Computer Systems, vol. 97, pp. 849-872, 2019.

[38] G. G. Wang, S. Deb, and L. S. Coelho, "Elephant Herding optimization," in Proceedings of the 2015 3rd International Symposium on Computational and Business Intelligence (ISCBI), pp. 1-5, IEEE, Bali, Indonesia, December 2015.

[39] S. Li, H. Chen, M. Wang, A. A. Heidari, and S. Mirjalili, "Slime mould algorithm: a new method for stochastic optimization," Future Generation Computer Systems, vol. 111, pp. 300-323, 2020. 